\title{
Effect of halloysite nanoclay on mechanical properties, thermal behavior and microstructure of cement mortars.
}

\begin{abstract}
Many studies have targeted the application of clay in cement composites and declared some enhancement on the properties of concrete. However there is little knowledge on nanoclays and their effect on the mechanical properties and durability of cement composites. Halloysite nanoclay is one of the subcategories of nanoclay that has been undeservedly ignored in the production of cement composites. Chemically, the outer surface of the halloysite nanotubes has properties similar to $\mathrm{SiO} 2$ while the inner cylinder core is related to $\mathrm{Al} 2 \mathrm{O} 3$ which together may improve the cement matrix. In this study the mechanical properties, flowability, thermal behavior and durability of mortars containing 1,2,3\% halloysite nanoclay were studied. Compressive strength and gas permeability of samples with $3 \%$ and $2 \%$ nanoclay were improved up to $24 \%$ and $56 \%$, respectively. SEM, XRD, DSC tests were carried out to investigate the microstructure and chemical composition change in samples with halloysite nanoclay.
\end{abstract}

Keyword: Halloysite nanoclay; Mechanical properties (C); Thermal analysis (B);

Microstructure (B); Mortar (E) 